\title{
Hybrid Indoor and Outdoor Tracking for Mobile 3D Mixed Reality
}

\author{
Wayne Piekarski, Ben Avery, Bruce H. Thomas, Pierre Malbezin \\ Wearable Computer Laboratory \\ School of Computer and Information Science \\ University of South Australia \\ Mawson Lakes, SA, 5095, Australia \\ wayne@cs.unisa.edu.au,avery@tinmith.net,bruce.thomas@unisa.edu.au,pierre@tinmith.net
}

\begin{abstract}
This paper describes a new hybrid tracking system that integrates standard outdoor augmented reality trackers with a low cost indoor tracker based on the use of fiducial markers. We use multiple cameras, separate orientation sensing, and scene graph integration to improve on similar previous systems. This hybrid tracker allows applications to operate within large indoor and outdoor environments with minimal scaling costs. Tracking is performed using GPS style world coordinates both indoors and outdoors, and has been integrated into our existing mobile $3 D$ modelling applications.
\end{abstract}

\section{Introduction}

We have been investigating for a number of years the use of mobile computers for performing augmented reality, mostly in outdoor environments. We initially presented the idea of a tracking system based on ARToolKit fiducial markers that is cheap to deploy and works indoors, where GPS currently does not work [4]. This tracking system was previously limited by the video capture and processing capabilities of the day, but is now realisable with the availability of powerful laptops and Firewire cameras.

This paper presents a new hybrid tracking system that uses existing outdoor tracking technology (such as GPS and orientation sensors) and combines these with a new indoor tracking system based on fiducial markers and the ARToolKit [2]. When the user walks around inside, multiple cameras on the backpack (see Figure 1) track the locations of markers in a room and provide absolute position information in real time with acceptable accuracy for our application. While the accuracy of our indoor tracking is not as good as other available commercial trackers, the required software and fiducial markers have negligible cost. This hybrid tracker allows our existing TinmithMetro modelling application [3] to be easily extended from outdoor only operation to simple indoor use as well.

In a similar spirit to our roof tracker developments, Kalkusch et al [1] have also performed research into the development of a tracking system using ARToolKit markers. Their tracker was developed to provide position and orientation information to a navigation system that acts as a guide for a user moving through a large building. While the navigation application has the capability to draw a wireframe model of the hallways being walked through, in most cases an arrow navigation cue and a hand held World-in-Miniature model are used to provide steering instructions for the user. This application does not need the more accurate and continuous tracking that is required for AR and VR systems where 3D objects must be accurately registered with the physical world. Our work shares many common features with Kalkusch et al such as marker reuse and ARToolKit, but has improved accuracy.

\section{Features}

The hybrid tracking system described in this paper is based on the combination of tracking results from indoor and outdoor tracking engines. An integration object is used to monitor the results of both trackers and passes on the most up to date and accurate value using a simple switch. This simplified method works well since GPS tracking typically fails before the indoor tracking becomes active. For outdoor tracking, a Trimble Ag132 GPS measures position accurate to $50 \mathrm{~cm}$, and an InterSense IS-300 measures head orientation. When indoors, the GPS will

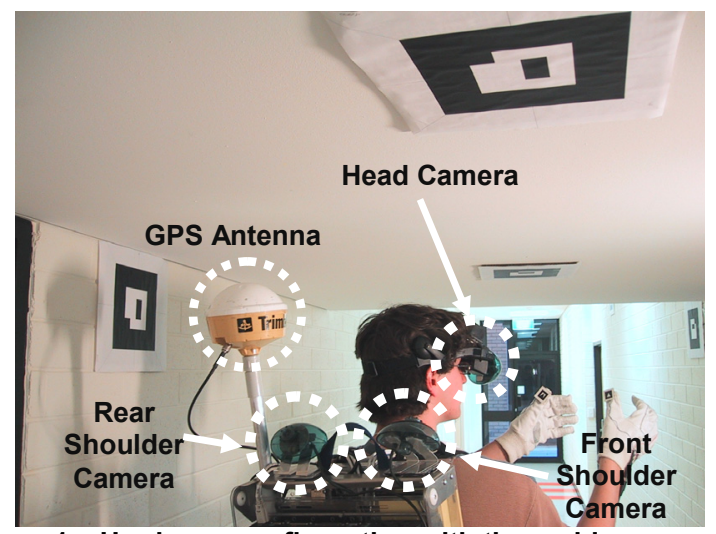

Figure 1 - Hardware configuration with three video cameras, GPS antenna, and fiducial markers on the hands and building

\begin{tabular}{|l|c|c|c|}
\hline & $X$ (east) & $Y($ north) & Z (height) \\
\hline Average & $9.8 \mathrm{~cm}$ & $10.2 \mathrm{~cm}$ & $19.7 \mathrm{~cm}$ \\
\hline Minimum & $1.0 \mathrm{~cm}$ & $4.5 \mathrm{~cm}$ & $15.5 \mathrm{~cm}$ \\
\hline Maximum & $17.5 \mathrm{~cm}$ & $19.0 \mathrm{~cm}$ & $24.0 \mathrm{~cm}$ \\
\hline
\end{tabular}

Figure 2 - Accuracy values for single rear shoulder camera 
not produce tracking results but the orientation sensor is still useable. The rest of this paper focuses on the implementation of the indoor tracking component.

For position tracking, the ARToolKit libraries are used to track fiducial markers placed on the ceiling and walls. While this library was designed to overlay 3D models onto fiducial markers, it can be used to perform tracking and so was used as a base for the tracker. This library is freely available making it ideal to use, although any other image tracking library with position and orientation extraction can also be used. Markers placed in the physical world are recorded using a scene graph model of the various rooms and buildings of interest, stored using GPS based world coordinates. When tracking results from ARToolKit are received, the scene graph calculates the world coordinates of the camera relative to the appropriate fiducial marker. Since the orientation values from ARToolKit are especially jittery and produce almost unusable AR overlays, the InterSense IS-300 is used to provide much more robust results at a very fast update rate. To integrate all the necessary tracking components, the Tinmith-evo5 software architecture and scene graph [3] are used to simplify the implementation of this tracker.

The indoor tracker is based on the use of fiducial markers that are $20 \mathrm{~cm}$ by $20 \mathrm{~cm}$, and placed on the ceilings and tops of walls of each room of interest. These markers are optimally placed to be visible to cameras elevated at 45 degrees and within 1-3 metres range, which produces the best quality tracking. Using cameras pointing directly upwards results in markers being closer to the user, but the close ceiling restricts the field of view and face on markers are poorly tracked by ARToolKit. Using a camera with a 30 degree field of view, we calculated that markers must be spaced approximately 2.5 metres apart so that a useable marker is usually visible during motion. When the user moves freely through the environment, we found that the tracking commonly failed because a single camera is not able to contain a visible useable marker at all times. To improve the probability of a visible marker, we use two shoulder mounted cameras (facing forwards and backwards at 45 degree elevation) as well as a forward looking camera also used for the video AR overlay. The front looking camera typically does not see markers since the user is focusing on their task and not the tracking, but since ARToolKit is already used for the existing hand tracking system, any marker detections can be used with no additional performance penalty. Greyscale 640x480 camera modes are used on the two shoulder cameras to conserve bandwidth on the Firewire 1394 bus, allowing the expansion of the system to more cameras if needed.

ARToolKit is limited to the number of marker patterns that can be searched for simultaneously, preventing the use of unique markers for an entire large building. We use a similar marker reuse strategy as Kalkusch et al [1], where a set of patterns are repeatedly used between rooms. Markers are placed on the doors leading between rooms to tell the system to switch between the room models in the scene graph. The tracking is expandable to a large number of rooms and hallways within buildings, with simple costs involved with the printing, placement, and entry of markers for the system to process. Marker patterns have been designed using black squares on a $4 \times 4$ grid that maps directly to ARToolKit's 16x16 sampling array, improving matching accuracy and reducing sampling errors compared to the use of human readable patterns. Since the output of the tracking results from ARToolKit are quite jittery and sometimes unreliable, simple averaging filters are used to smooth the results somewhat. Other heuristics such as the restriction of results to a maximum range and minimum time of marker gaze are also used to compensate for incorrect marker detection.

\section{Conclusion}

We performed some simple informal experiments to measure the accuracy of the tracker when in use indoors. Using a single shoulder mounted camera, the accuracy of the tracker was measured from ten randomly selected locations where a single marker was visible. The results of these tests are summarised in Figure 2 and indicate typical errors between 10 to 20 centimetres in each axis. When multiple markers are in use with averaging then these results may be improved further, but this is difficult to measure accurately.

The hybrid tracker summarised in this paper is far from perfect and suffers from a number of problems that will be addressed in further research. Some current problems are dealing with the transition between indoor to outdoor, false marker detection, varying lighting conditions, and achieving continuous tracking of at least one marker. Given these limitations, the tracker is still useful and has been integrated into our existing Tinmith-Metro modelling application to allow both indoor and outdoor operation.

\section{References}

[1] Kalkusch, M., Lidy, T., Knapp, M., Reitmayr, G., Kaufmann, H., and Schmalstieg, D. Structured Visual Markers for Indoor Pathfinding. In 1st Int'l Augmented Reality Toolkit Workshop, Darmstadt, Germany, Sep 2002.

[2] Kato, H. and Billinghurst, M. Marker Tracking and HMD Calibration for a Video-based Augmented Reality Conferencing System. In 2nd Int'l Workshop on Augmented Reality, pp 85-94, San Francisco, Ca, Oct 1999.

[3] Piekarski, W. and Thomas, B. H. An Object-Oriented Software Architecture for 3D Mixed Reality Applications. In 2nd Int'l Symposium on Mixed and Augmented Reality, Tokyo, Japan, Oct 2003.

[4] Thomas, B., Close, B., Donoghue, J., Squires, J., De Bondi, P., Morris, M., and Piekarski, W. ARQuake: An Outdoor/Indoor Augmented Reality First Person Application. In 4th Int'l Symposium on Wearable Computers, pp 139146, Atlanta, Ga, Oct 2000. 